\title{
Evolución de las licencias federativas de pádel en Andalucía (2009-2016)
}

\section{Evolution of paddle federal licenses in Andalucía (2009-2016)}

\author{
Nicolás Fernández-Martínez ${ }^{1}$, Ramón Gómez-Chacón ${ }^{1}$, Ainara Bernal-García ${ }^{2}$ y Marta Pérez-Villalba ${ }^{3}$ \\ 1 CEU San Pablo Andalucía. \\ 2 Agencia para la Gestión, Investigación e Innovación en Servicios Deportivos (AGIISD). \\ 3 Centro Universitario San Isidoro. Sevilla.
}

\begin{abstract}
Resumen: Los últimos años del deporte en Espańa muestran un alto crecimiento en la práctica del pádel (Gómez-Chacón, Pascua-Barón y Fernández-Martínez, 2017). El pádel, es el deporte de raqueta más practicado semanalmente por la población Española(Consejo Superior de Deportes, 2015).Asimismo, y siguiendo la línea de otros autores, el pádel, es el deporte que más se ha desarrollado en los últimos 23 años con un crecimiento del 1.947,41\% (López, 2017).El objetivo de este estudio es conocer la evolución de las licencias federativas de pádel en Andalucía durante los ańos 2009-2016, para poder certificar que todos los datos encontrados coinciden con lo que han afirmado diversos autores y organismos públicos. Se consultó la base de datos de la federación Andaluza de Pádel. Todos los datos extraídos en la bibliografía consultada no hacen más que indicar que el pádel es uno de los deportes más emergentes y con mayor crecimiento y evolución del siglo XXI.
\end{abstract}

Palabras clave. Pádel, licencia federativa, categoría absoluta, Andalucía, evolución.
Abstract: The last years of sport in Spain show a high growth in the practice of paddle (Gómez-Chacón, Pascua-Barón and Fernández-Martínez, 2017). The paddle, is the sport of racket more practiced weekly by the Spanish population (Superior Council of Sports, 2015). Likewise, and following the line of other authors, paddle is the sport that has most developed in the last 23 years with a growth of $1,947.41 \%$ (López, 2017). The objective of this study is to know the evolution of the federative paddle licenses in Andalusia in absolute category during the years 2009-2016 in order to certify that all the data found coincide with what various authors and public bodies have affirmed. The database of the Andalusian federation of Paddle was consulted. All the data extracted in the consulted bibliography do not do more than indicate that the paddle is one of the most emergent sports and with greater growth of the XXI century.

Keywords. Paddle, federative license, absolute category, Andalusia, evolution.

\section{Introducción}

Los últimos años del deporte en España muestran un alto crecimiento en la práctica del pádel. El pádel es considerado por Sánchez-Alcaraz (2013) como un deporte joven. Se puede observar un amplio colectivo formado por personas jóvenes y mayores que cada vez ayudan más al desarrollo de este deporte (Gómez-Chacón, Pascua-Barón, yFernándezMartínez, 2017).

En cuanto a los deportes de raqueta, a nivel de práctica deportiva se observa como el pádel ha tenido un aumento notable desde 2010 a 2015, doblando casi su número de practicantes semanales (Consejo Superior de Deportes, 2015). Otros autores destacan que el pádel durante los ańos 2005 a 2010 ha sufrido un crecimiento del 300\% (García Ferrando y Llopis-Goig, 2011).

Este gran incremento lo justifican los autores por la facilidad de su práctica en las fases iniciales, por las características de la pista, por la continuidad que le da al juego tener paredes que retengan la pelota en el interior de la cancha, además de por la baja exigencia fisiológica (Lasaga, 2011; Sánchez-Alcaraz, 2014).

Dirección para correspondencia [Correspodence address]: Nicolás Fernández-Martínez. CEU San Pablo Andalucía. (España). E-mail: nfernandez@ceuandalucia.es
El tenis $(1,9 \%)$ y el pádel $(3,7 \%)$, son los deportes de raqueta más practicados semanalmente por la población Española aunque están muy por debajo de otros como la gimnasia, carrera o el ciclismo (Consejo Superior de Deportes, 2015).

A día de hoy, existe poca literatura científica relacionada con el pádel (Sánchez-Alcaraz, 2013; Courel, SánchezAlcaraz, y Cañas, 2014). En el 2015 se publicó un artículo sobre las investigaciones científicas encontradas en torno al pádel, la mayoría de estos trabajos (84,3\%) estaban escritos en castellano, el $27.4 \%$ la temática estaba relacionada con el análisis del juego y en un $42 \%$ se utilizaron como muestra a jugadores profesionales (Sánchez-Alcaraz, Cañas, y CourelIbáńez, 2015).

El pádel ha evolucionado en cuanto a práctica deportiva semanal, duplicándose durante el periodo 2010-2015 (de $1,7 \%$ a 3,7\%). El frontón y frontenis (de 0,5\% a 0,9\%) y por otra parte, el tenis no ha visto incrementada su práctica durante este mismo periodo (de 1,9\% a 1,9\%) (Figura 1). 


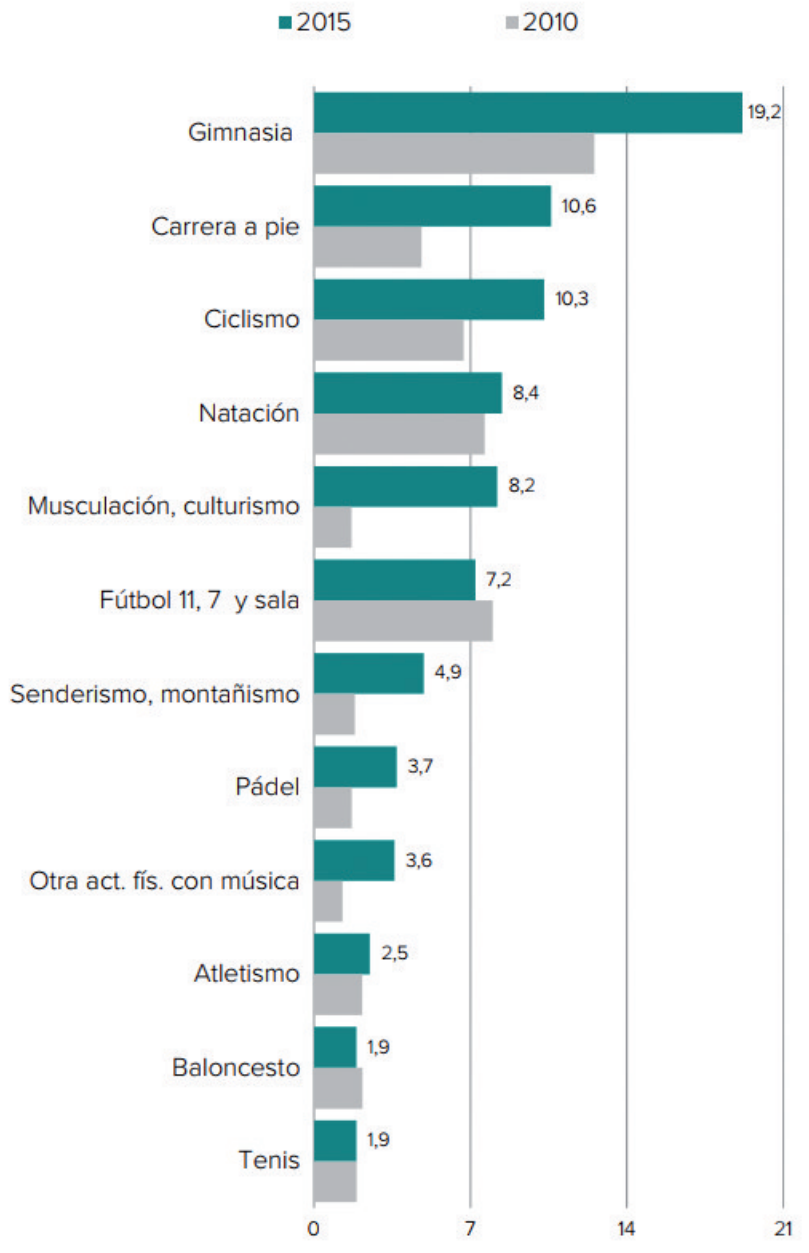

Figura 1. Personas que practican deportes semanalmente según las modalidades deportivas más frecuentes (Fuente: Consejo Superior de Deportes, 2015).

Los datos encontrados revelan que el Pádel es el deporte más practicado por los españoles durante el año 2015 (16,8\%), seguido del tenis (14\%), Ping-pong $(11,1 \%)$, frontón y frontenis $(6,6 \%)$ y finalmente el squash $(1,9 \%)$. No aparece ningún dato relacionado con el bádminton (Tabla 1).

Tabla 1. Personas que practicaron deporte en el 2015 según modalidad (\% de la población que practicó deporte en el último año de cada colectivo) (Fuente: Consejo Superior de Deportes, 2015).

\begin{tabular}{lccc}
\hline & TOTAL & HOMBRES & MUJERES \\
\hline TOTAL & 100 & 100 & 100 \\
Frontón, frontenis & 6,6 & 9 & 3,6 \\
Tenis & 14 & 18,1 & 9,1 \\
Ping-pong & 11,1 & 14,1 & 7,2 \\
Pádel & 16,8 & 21,7 & 11 \\
Squash & 1,9 & 2,6 & 1,1 \\
\hline
\end{tabular}

En cuanto a la disponibilidad de raquetas para la práctica deportiva, el $54,1 \%$ de la población posee algún tipo de raqueta en su hogar (Figura 2). Las raquetas es el tercer equipamiento deportivo más adquirido por la población después de las bicicletas (63\%) y los balones (59,3\%).

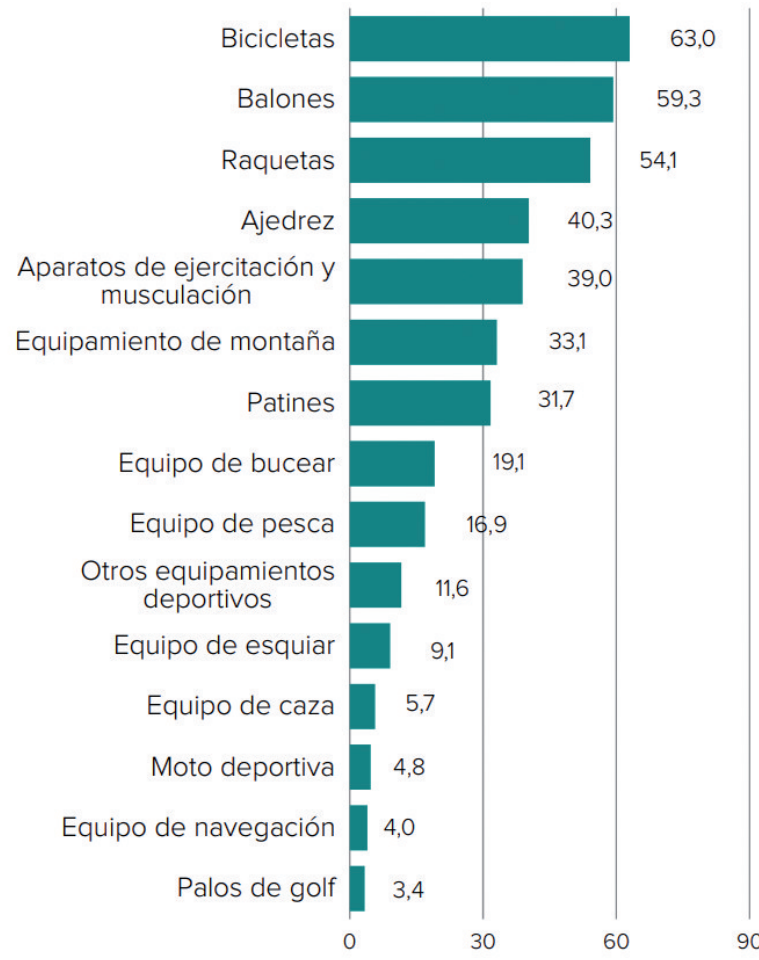

Figura 2. Personas que disponen de equipamientos deportivos en el hogar según tipo (Fuente: Consejo Superior de Deportes, 2015).

En la Tabla 2 se puede observar que las raquetas de tenis $(35,3 \%)$ es el implemento más adquirido por las personas para su hogar, seguido de las palas de ping-pong (22\%) y las palas de pádel (21,9\%).

Tabla 2. Personas según la disponibilidad de diversos equipamientos deportivos en su hogar (\% de la población de cada colectivo) (Fuente: Consejo Superior de Deportes, 2015).

\begin{tabular}{lcc}
\hline & $\begin{array}{c}\text { \% Población } \\
\text { Total }\end{array}$ & $\begin{array}{c}\text { En porcentaje de la población que } \\
\text { practicó deporte en el último año }\end{array}$ \\
\hline TOTAL & 100 & 100 \\
Raquetas: & 54,1 & 69 \\
Palas de pádel & 21,9 & 31,2 \\
Tenis & 35,3 & 45,9 \\
Ping pong & 22 & 29,6 \\
Otras & 27,2 & 36,2 \\
\hline
\end{tabular}

La frecuencia con que los espańoles practican los deportes de raqueta se detalla en la Tabla 3. El pádel lo practican una 
vez al año el 9\% de la población analizada en este estudio, seguido del tenis con 7,5\% y el frontón y frontenis con 3,5\%.
Al menos una vez a la semana el pádel lo practica el 3,7\% y el tenis el 1,9\% (Consejo Superior de Deportes, 2015).

Tabla 3. Personas según la frecuencia de la práctica deportiva por modalidad deportiva (\% de la población total analizada) (Fuente: Consejo Superior de Deportes, 2015).

\begin{tabular}{lccc}
\hline & Al menos 1 vez al año & Al menos 1 vez a la semana & Al menos 1 vez al mes \\
\hline TOTAL (miles) & 38.934 & 38.934 & 38.934 \\
$\%$ & $100 \%$ & $100 \%$ & $100 \%$ \\
Total practican (\%) & $53,5 \%$ & $46,2 \%$ & $51 \%$ \\
Frontón, frontenis & 3,5 & 0,9 & 2 \\
Tenis & 7,5 & 1,9 & 4,6 \\
Ping-pong & 6 & 1,1 & 3,2 \\
Pádel & 9 & 3,7 & 6,6 \\
Squash & 1 & 0,3 & 0,6 \\
\hline
\end{tabular}

En cuanto a los indicadores de práctica deportiva, existe una tabla (Tabla 4), que indica la relación existente entre diversos deportes y los deportes de raqueta. Cabe destacar que las personas que practican baloncesto también hacen pádel
$(34,7 \%)$, que las que practican fútbol-sala también hacen pádel $(34,2 \%)$ y las que practican fútbol y fútbol 7 , también hacen pádel $(30,5 \%)$.

Tabla 4. Indicadores de práctica deportiva anual en la población total analizada y en la población que practica ciclismo, natación, fútbol 11 y 7 , fútbol sala y baloncesto (\% del total vertical) (Fuente: Consejo Superior de Deportes, 2015).

\begin{tabular}{lcccccc}
\hline & $\begin{array}{c}\text { \% Población Total } \\
\text { Investigada }\end{array}$ & $\begin{array}{c}\text { \% que practican } \\
\text { ciclismo }\end{array}$ & $\begin{array}{c}\text { \% que practican } \\
\text { natación }\end{array}$ & $\begin{array}{c}\text { \% que practican } \\
\text { fútbol 11 y 7 }\end{array}$ & $\begin{array}{c}\text { \% que practican } \\
\text { fútbol sala }\end{array}$ & $\begin{array}{c}\text { que practican } \\
\text { baloncesto }\end{array}$ \\
\hline TOTAL \% & 100 & 100 & 100 & 100 & 100 & 100 \\
Frontón, frontenis & 3,5 & 12 & 10,6 & 14 & 18,7 & 21,9 \\
Tenis & 7,5 & 22,5 & 21,4 & 29,6 & 36,2 & 40,7 \\
Ping-pong & 6 & 19,5 & 18,5 & 25,2 & 31,7 & 36,9 \\
Pádel & 9 & 23,5 & 22,5 & 30,5 & 34,2 & 34,7 \\
Squash & 1 & 3 & 3,1 & 4,2 & 5,5 & 6,3 \\
\hline
\end{tabular}

Por otra parte, es importante destacar que el 38\% de la población que practica tenis también realiza pádel. Los mismo ocurre con el frontón y frontenis (16\%), ping-pong (27,3\%), y squash (7,7\%). Existe un dato destacable, que indica que el 45,7 \% de los jugadores que practican pádel, también hacen tenis.

Por lo que el objetivo principal de este trabajo es conocer la evolución de las licencias federativas de pádel en Andalucía en categoría absoluta durante los años 2009-2016, y a su vez comparar la evolución de la categoría absoluta con las categorías de menores y veteranos.

\section{Método}

\section{Participantes e instrumentos}

Para extraer los datos sobre el número de licencias federativas de pádel a nivel andaluz se recurrió por un lado a los ficheros presentados por el Consejo Superior de Deportes (Consejo Superior de Deportes, 2016). Estos ficheros recogen datos estadísticos correspondientes al número de licencias y clubes federados de las diferentes Federaciones Españolas, a nivel estatal y nivel autonómico, desde 1994 hasta 2016. Por otro lado, se extrajeron datos sobre las licencias federativas a la categoría absoluta de pádel en referencia al género y a las provincias de Andalucía entre los años 2009-2016 (Federación Andaluza de Pádel, 2017).

\section{Análisis de datos}

Se realizó análisis descriptivos (frecuencias y porcentajes) además de tablas cruzadas entre las variables licencias federativas, categoría absoluta, género y provincias de Andalucía. Con los análisis descriptivos de los datos se realizaron diferentes figuras a través de una hoja de 
cálculo mediante el programa Microsoft Excel 2010 para Windows.

\section{Resultados}

El número de licencias federativas en Andalucía en la categoría absoluta refleja un disminución del año 2009 al 2012, perdiendo 258 licencias federativas, sin embargo en el año 2013 se produce un aumento considerable de las licencias federativas recuperando en un ańo (255 licencias federativas) las que se perdieron durante los cuatro años anteriores, además en 2014 se produce el hecho más destacable con un incremento de 500 licencias federativas y sigue incrementándose de manera más paulatina en 2015 y 2016 (Figura 3).

En la mayoría de las provincias andaluzas se ha producido un aumento considerable del número de licencias federativas en pádel entre 2009-2016 destacando Granada con 147 licencias en 2009 a 372 licencias en 2016, y Jaén con 103 licencias en 2009 a 360 licencias en 2016.

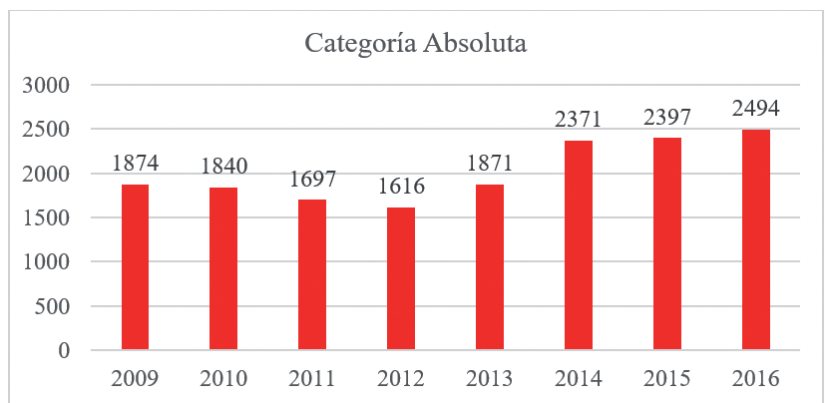

Figura 3. Número de licencias categoría absoluta en Andalucía 2009-2016.

Por otro lado, destaca la evolución de licencias federativas en Cádiz, ya que han ido disminuyendo paulatinamente de 412 licencias en 2009 a 209 en 2016. Otro hecho destacable es la disminución en Córdoba de 80 licencias de 2014 a 2015, y la recuperación de dichas licencias (81 licencias) en 2016 (Figura 4).

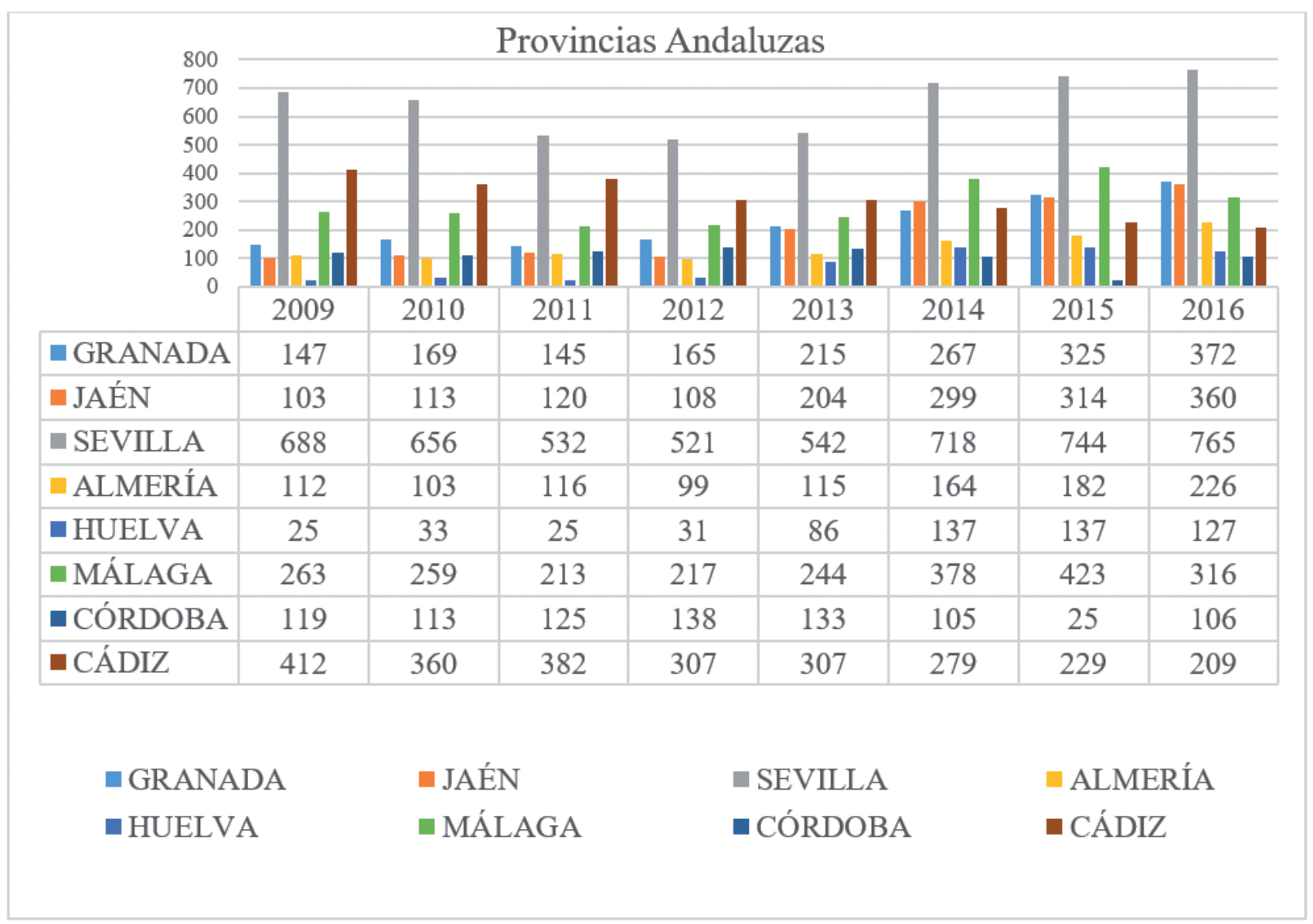

Figura 4. Número de licencias categoría absoluta en las provincias andaluzas 2009-2016. 
En relación con el número de licencias por género cabe destacar que ambos sexos se ha producido un incremento de licencias, no obstante, la evolución ha sido mayor en hombres que en mujeres pasando en el género masculino de 1208 licencias en 2009 a 1711 en 2016, y en el género femenino de 666 licencias en 2009 a 783 en 2016 (Figura 5).

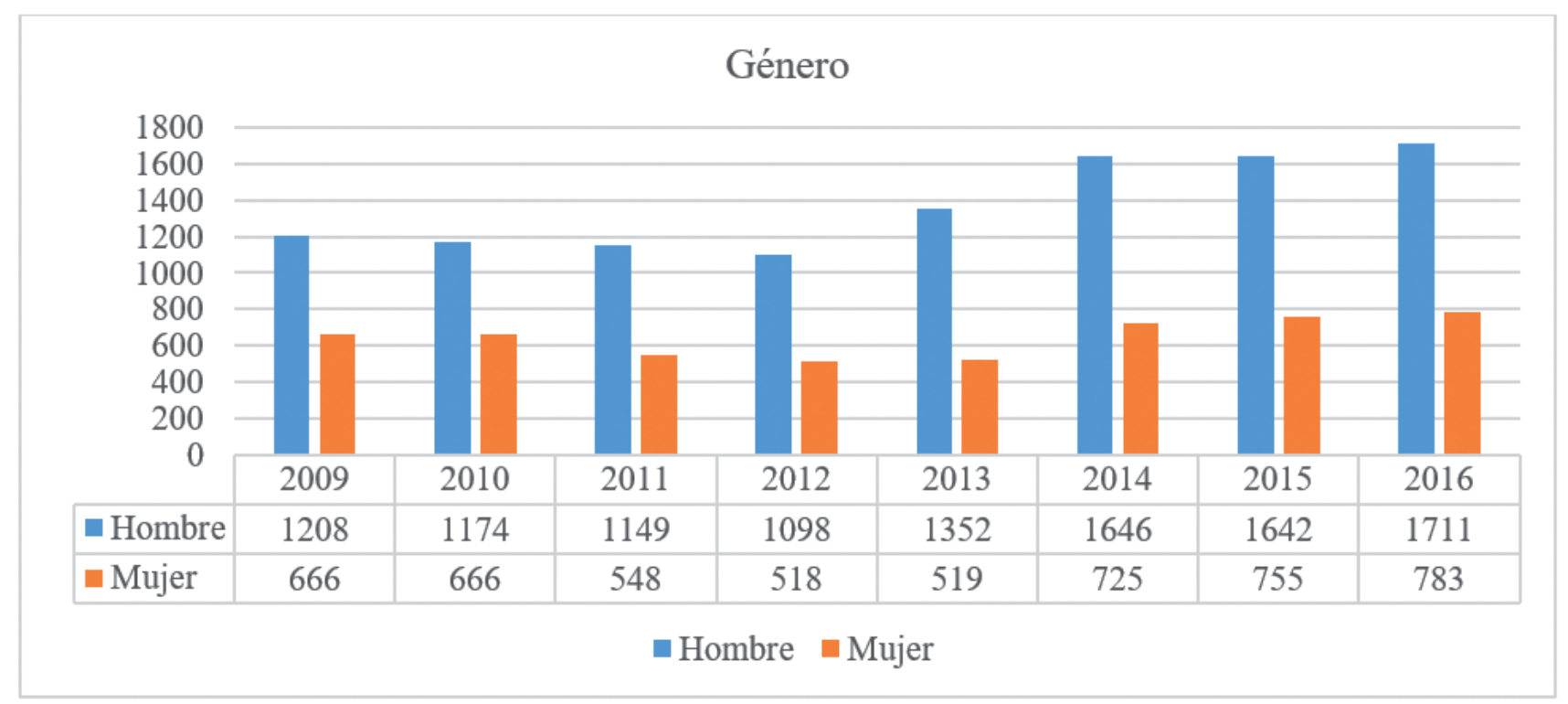

Figura 5. Número de licencias categoría absoluta por género 2009-2016.

Además, en la tabla 5, se puede observar cómo evolucionan desde el año 2009 hasta el año 2016 las tres grandes categorías existentes en el pádel español y en este caso en el andaluz, categoría de menores, absoluta y veterana.

En el año 2009 existía un 4\% de menores que tenían licencia federativa, un $26,4 \%$ de jugadores absolutos y un $69,5 \%$ de veteranos, siendo esta última categoría la más representativa del pádel andaluz. Conviene subrayar que, a lo largo de los años estudiados, los menores han visto aumentada su participación en un 10,6\%, sin embargo, en la absoluta no se aprecian cambios significativos perdiendo tan solo $1,2 \%$ de representatividad dentro de la federación andaluza, sin embargo, los veteranos han perdido un $8,7 \%$.
Tabla 5. Porcentaje de licencias federativas en las categorías de menores, absolutas y veteranas (Fuente: Federación Andaluza de Pádel, 2017).

\begin{tabular}{cccc}
\hline \multicolumn{3}{c}{ CATEGORIA \% } \\
\hline AÑO & MENORES & ABSOLUTO & VETERANO \\
\hline 2009 & 4 & 26,4 & 69,5 \\
\hline 2010 & 5,0 & 25,8 & 69,2 \\
\hline 2011 & 7,2 & 24,6 & 68,2 \\
\hline 2012 & 8,8 & 23,7 & 67,5 \\
\hline 2013 & 11,7 & 25,4 & 62,9 \\
\hline 2014 & 14,0 & 25,2 & 60,8 \\
\hline 2015 & 14,6 & 25,2 & 60,2 \\
\hline 2016 & 16,5 & 25,4 & 58,1 \\
\hline
\end{tabular}

En esta figura (Figura 6), donde se pueden ver datos cruzados de provincia y categorías, se detallan con mayor claridad como las licencias de menores aumentan su protagonismo dentro de la federación (color azul) y las licencias de veteranos lo disminuyen (color gris). 


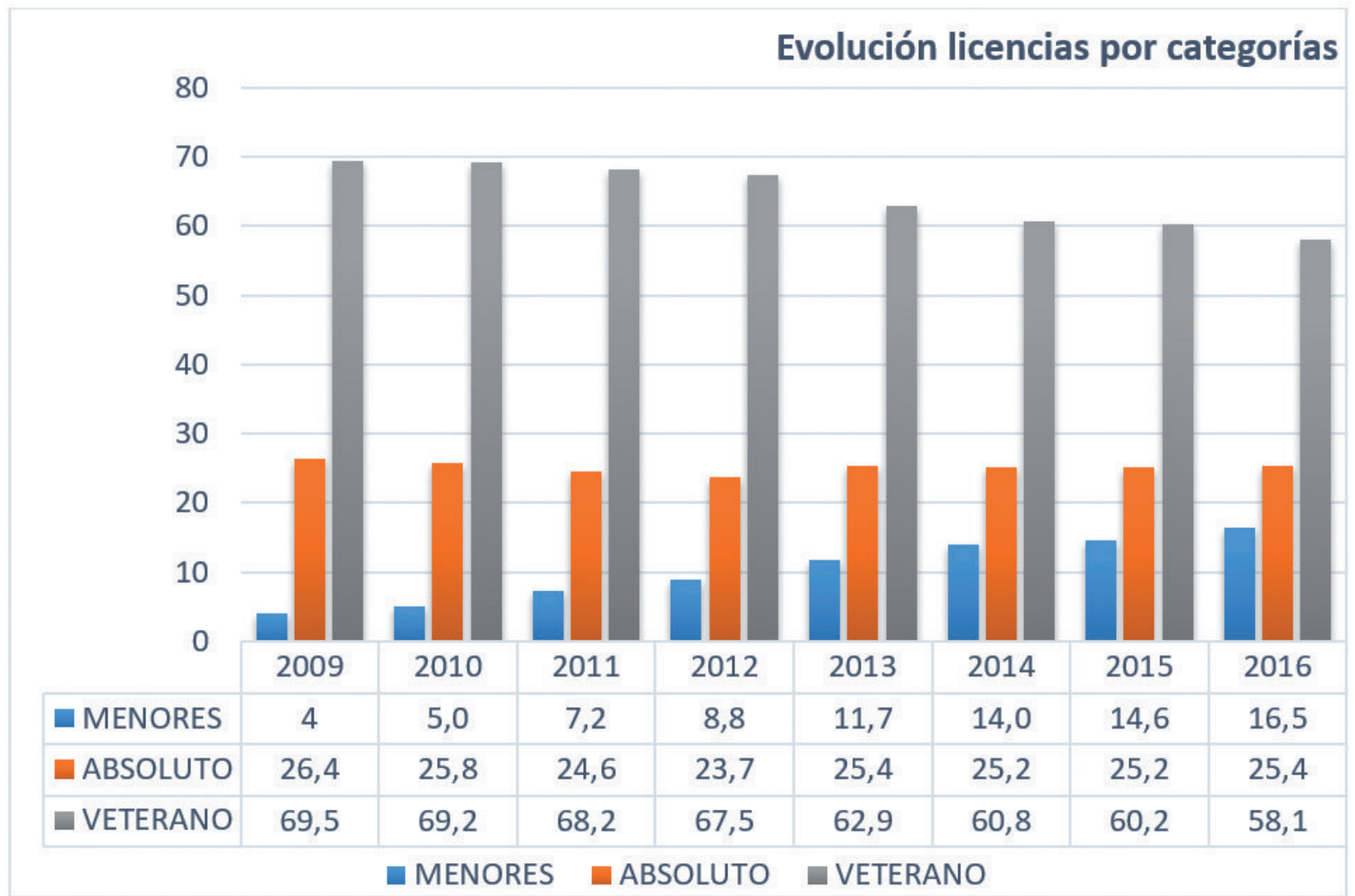

Figura 6. Porcentaje de licencias federativas en las categorías de menores, absolutos y veteranos durante los años 2009 a 2016 (Fuente: Federación Andaluza de Pádel, 2017).

En la tabla cruzada que se expone a continuación (Tabla 6), se puede ver con detalle el número de licencias federativas existentes en cada provincia durante todos los ańos estudiados. Al igual que en la figura 4, la provincia de Cádiz también va perdiendo paulatinamente licencias tanto en la categoría absoluta y de veteranos, sin embargo, cabe destacar que la categoría de menores sigue una línea ascendente de crecimiento. La provincia de Córdoba tiene un crecimiento ascendente en la categoría de menores, sin embargo, solo y exclusivamente en el año 2015, tiene una gran pérdida de licencias y se recupera durante el ańo 2016. La provincia de Huelva no tiene licencias federativas de menores durante los ańos 2009-2011, en el 2012 aparece con una licencia, en el 2013 con 19 licencias y finalizando el ańo 2016 con 95 licencias. En todas las demás provincias de Andalucía no se aprecian cambios significativos a destacar.

Tabla 6. Número de licencias federativas por provincia en las categorías de menores, absolutos y veteranos(Fuente: Federación Andaluza de Pádel, 2017).

\begin{tabular}{|c|c|c|c|c|c|c|c|c|c|}
\hline & & GRANADA & JAÉN & SEVILLA & ALMERÍA & HUELVA & MÁLAGA & CÓRDOBA & CÁDIZ \\
\hline \multirow{3}{*}{2009} & MEN & 49 & 12 & 84 & 12 & 0 & 14 & 64 & 52 \\
\hline & ABS & 147 & 103 & 688 & 112 & 25 & 263 & 119 & 412 \\
\hline & VET & 588 & 456 & 1451 & 482 & 80 & 784 & 151 & 938 \\
\hline \multirow{3}{*}{2010} & MEN & 63 & 9 & 102 & 13 & 0 & 27 & 76 & 65 \\
\hline & ABS & 169 & 113 & 656 & 103 & 33 & 259 & 113 & 360 \\
\hline & VET & 598 & 507 & 1464 & 477 & 69 & 767 & 142 & 857 \\
\hline \multirow{3}{*}{2011} & MEN & 63 & 8 & 162 & 10 & 0 & 68 & 86 & 101 \\
\hline & ABS & 145 & 120 & 532 & 116 & 25 & 213 & 125 & 382 \\
\hline & VET & 542 & 457 & 1419 & 477 & 82 & 707 & 149 & 820 \\
\hline
\end{tabular}




\begin{tabular}{|c|c|c|c|c|c|c|c|c|c|}
\hline & & GRANADA & JAÉN & SEVILLA & ALMERÍA & HUELVA & MÁLAGA & CÓRDOBA & CÁDIZ \\
\hline \multirow{3}{*}{2012} & MEN & 67 & 10 & 194 & 21 & 1 & 90 & 113 & 107 \\
\hline & $\mathrm{ABS}$ & 165 & 108 & 521 & 99 & 31 & 217 & 138 & 307 \\
\hline & VET & 589 & 424 & 1533 & 403 & 65 & 674 & 178 & 694 \\
\hline \multirow{3}{*}{2013} & MEN & 61 & 36 & 232 & 44 & 19 & 128 & 156 & 190 \\
\hline & ABS & 215 & 204 & 542 & 115 & 86 & 244 & 133 & 307 \\
\hline & VET & 588 & 476 & 1532 & 346 & 204 & 730 & 193 & 537 \\
\hline \multirow{3}{*}{2014} & MEN & 124 & 73 & 366 & 47 & 43 & 233 & 192 & 231 \\
\hline & ABS & 267 & 299 & 718 & 164 & 137 & 378 & 105 & 279 \\
\hline & VET & 777 & 607 & 1824 & 492 & 283 & 1016 & 191 & 485 \\
\hline \multirow{3}{*}{2015} & MEN & 135 & 77 & 406 & 54 & 92 & 335 & 50 & 240 \\
\hline & ABS & 325 & 314 & 744 & 182 & 137 & 423 & 25 & 229 \\
\hline & VET & 842 & 632 & 1924 & 479 & 325 & 1062 & 56 & 399 \\
\hline \multirow{3}{*}{2016} & MEN & 151 & 138 & 502 & 76 & 95 & 242 & 149 & 258 \\
\hline & $\mathrm{ABS}$ & 372 & 360 & 765 & 226 & 127 & 316 & 106 & 209 \\
\hline & VET & 874 & 639 & 1991 & 451 & 287 & 903 & 186 & 350 \\
\hline
\end{tabular}

\section{Discusión y conclusiones}

En este estudio se ha realizado un amplio análisis de la evolución de las licencias federativas de pádel desde el 2009 hasta el año 2016. Los resultados de esta investigación confirman que el pádel sigue en estado de continuo crecimiento. Tanto las licencias federativas absolutas como de menores han aumentado, sin embargo las licencias de veteranos han disminuido, aunque en el cómputo global de licencias, el pádel en Andalucía sigue en creciendo año tras año. Cabe destacar que durante los años 2011 y 2012, se produce un descenso en las licencias deportivas, y coincide con el ańo olímpico y por tanto con elecciones federativas autonómicas. Durante estos años se produjeron cambios en la presidencia de la federación. Es habitual encontrar descensos de licencias federativas en otras federaciones cuando se producen cambios en los cargos directivos de este tipo de instituciones. Es por ello, por lo que estos datos pueden reflejar este decrecimiento.Sevilla, Granada, Jaén y Málaga por este orden, son las provincias con mayor número de licencias en el año 2016.

El pádel en categoría de menores ha aumentado su cuota de participación. Cada vez son más los menores que sacan su licencia federativa para participar en los eventos que este ente organiza y se puede observar el crecimiento al alza en los últimos ańos. Este cambio puede venir por el gran impulso, por la cantidad y calidad de las competiciones, por el interés de los clubes de organizar actividades para este colectivo, debido al gran público que arrastra, y por muchos otros factores que podrían ser estudiados en el futuro.

El pádel en categoría de veteranos pierde un gran porcentaje de licencias federativas durante los años analizados. Esto puede ser debido a la poca calidad de las competiciones, al escaso número de eventos y actividades destinados a este colectivo, a los insuficientes premios en metálico o en especies que los clubes o federación destinan a ellos y por supuesto, otros muchos factores que también podrían ser estudiados. El colectivo de mayor representatividad en la federación andaluza de pádel (58,1\% en el año 2016-veteranos), debería ser un colectivo con una gran satisfacción a nivel de usuario, y que continuara ayudando al crecimiento de esta organización.

La Federación Andaluza de pádel, tendría que plantearse una oferta de servicios deportivos más atractivos para que el pádel femenino en Andalucía pueda tener un crecimiento similar al masculino.

\section{Limitaciones y futuras líneas de investigación}

La principal limitación de este estudio fue el acceso a la base de datos de la Federación Andaluza de Pádel para obtener los datos. Las federaciones deportivas tienen carácter de entidades privadas, aunque también un carácter mixto, es decir, ejercen por delegación, funciones públicas de carácter administrativo, actuando en este caso como agentes de la Administración Pública. Por lo tanto, aunque los ficheros de las Federaciones deportivas que se crean para el ejercicio de las funciones públicas de carácter administrativo son de titularidad pública (ficheros de federados, competiciones y resultados, controles de salud y de dopaje y sanciones), existen otros ficheros que respondan a actividades propias de las federaciones deportivas, como el de empleados, o proveedores, que serán de titularidad privada. A pesar de que 
todo esto está regulado y legislado, es difícil el acceso a esos datos.

En cuanto a las futuras líneas de investigación, sería importante investigar la evolución de las licencias federativas en las categorías inferiores y categorías de veteranos, así como la evolución de licencias federativas por categorías y por comunidades autónomas.

Otra línea de investigación sería, profundizar las causas que provocan el aumento paulatino de licencias federativas en categoría de menores y la disminución en la categoría de veteranos.
El conocimiento de la evolución de las licencias federativas en el pádel está estrechamente relacionado con las implicaciones para la gestión, de modo que las organizaciones, tanto públicas como privadas, pueden administrar y organizar sus instalaciones deportivas para diferentes eventos profesionales y amateur y las empresas de equipamientos y materiales deportivos puedan gestionar y organizar sus recursos en función de la evolución y de la tendencia de las diferentes modalidades deportivas de los deportes de raqueta, en este caso más concreto del pádel.

\section{Referencias}

1. Barbero, G. (2007). Didáctica de una clase de pádel. Retos. Nuevas tendencias en Educación Física, Deporte y Recreación, (12).

2. Consejo Superior de Deportes (2016). Federaciones Deportivas Españolas. Licencias. Recuperado de: http://www.csd.gob.es/csd/ asociaciones/1fedagclub/03Lic

3. Consejo Superior de Deportes. (2015). Encuesta de hábitos deportivos 2015. Recuperado de https://www.mecd.gob.es/servicios-al-ciudadano$\mathrm{mecd} / \mathrm{dms} / \mathrm{mecd} /$ servicios-al-ciudadano-mecd/estadisticas/deporte/ ehd/Encuesta_de_Habitos_Deportivos_2015.pdf

4. Federación Andaluza de Pádel (2017). Base de datos de licencias deportivas. Recuperado de www.fap.es.

5. Federación Española de Pádel (2017). Federaciones y delegaciones autonómicas. Recuperado de: http://www.padelfederacion.es/ Federaciones.

6. Federación Española de Tenis (2017). Federaciones y delegaciones autonómicas. Recuperado de http://www.rfet.es/.

7. García-Ferrando, M. y Llopis-Goig, R. (2011). Ideal democrático y bienestar personal. Los hábitos deportivos en España 2010. Madrid: Consejo Superior de Deportes y Centro de Investigaciones Sociológicas.

8. Gómez-Chacón, R., Pascua-Barón, D., Fernández-Martínez, N. (2018) Evolución de las licencias federativas (1994-2016). Pádel vs Tenis. Materiales para la historia del deporte, 16, 43-49.

9. Lasaga, M. J. (2011). Estudio social y metodológico del pádel desde la percepción de técnicos y jugadores: una apuesta educativa. Tesis Doctoral. Universidad de Sevilla.

10. López, P. (2017). Del fútbol al pádel: ¿Que deportes han crecido más en federados desde Barcelona 1992? Palco 23. Recuperado de: https:// www.palco23.com/entorno/del-futbol-al-padel-que-deportes-hancrecido-mas-en-federados-desde-barcelona-1992.html

11. Madison. (2013). El desarrollo del pádel en España a través de clubes y practicante de pádel. II Congreso Internacional de la industria del pádel. Madrid.

12. Ministerio de Educación, Cultura y Deporte (2017). Anuario de Estadísticas Deportivas 2017. Recuperado de: https:/www.mecd.gob. es/servicios-al-ciudadano- $\mathrm{mecd} / \mathrm{dms} / \mathrm{mecd} /$ servicios-al-ciudadano$\mathrm{mecd} /$ estadisticas/deporte/anuario-deporte/AED-2017/Anuario_de_ Estadisticas_Deportivas_2017.pdf.

13. Sánchez-Alcaraz, B. J., Cañas, J., \&Courel-Ibáñez, J. (2015). Análisis de la investigación científica en pádel. AGON Revista International Journal of Sport Sciences, 5(1), 44-54.

14. Sánchez-Alcaraz, B.J. (2013). Historia del pádel. Materiales para la historia del deporte, 11, 57-60.

15. Sánchez-Alcaraz, B.J. (2014). Diferencias en las acciones de juego y la estructura temporal entre el pádel masculino y femenino profesional. Acción motriz, 12, 17-22. 